\title{
Covid-19-Schutzmaßnahmen und Einschränkungen des sozialen Lebens in Pflegeheimen
}

\author{
Analyse von Verordnungen und Surveydaten \\ Annabell Gangnus ${ }^{1}$, Christian Hering ${ }^{1}$, Raphael Kohl' \\ Clara Sophia Henson ${ }^{1}$, Antje Schwinger², Elisabeth Steinhagen-Thiessen ${ }^{3}$, \\ Adelheid Kuhlmey ${ }^{1}$, Paul Gellert ${ }^{1}$ \\ ${ }^{1}$ Institut für Medizinische Soziologie und Rehabilitationswissenschaft, Charité - Universitätsmedizin Berlin \\ ${ }^{2}$ Forschungsinstitut der kommunalen Krankenkassen (WIdO), Berlin \\ ${ }^{3}$ Abteilung für Endokrinologie und Stoffwechsel, Charité - Universitätsmedizin Berlin
}

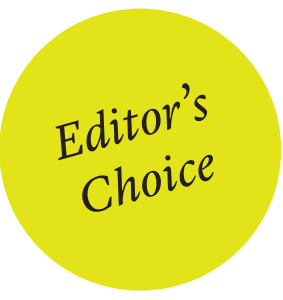

\begin{abstract}
Zusammenfassung: Hintergrund: Das Spannungsfeld zwischen Gesundheitsschutz und Einschränkungen des sozialen Lebens in den Pflegeheimen während der Pandemie ist unzureichend aufgearbeitet. Ziel: Ziel war es, bundesländerspezifische Verordnungen zu systematisieren und die Umsetzungen dieser in einen Zusammenhang mit Einschränkungen sozialer Angebote für die Bewohner_innen zu bringen. Methoden: In einem Mixed-Methods-Design wurden Schutzmaßnahmen für Pflegeheime in der ersten Pandemiewelle aus 450 Verordnungen kategorisiert, in ein theoretisches Maßnahmenmodell eingespeist und in einem Survey unter Heimleitungen ( $n=1260$ ) zur Handhabung der Maßnahmen sowie zum Wegfall sozialer Angebote verwendet. Der Zusammenhang von Schutzmaßnahmen und Einschränkungen sozialer Angebote wurde mit binärlogistischen verallgemeinerten Schätzgleichungen analysiert. Ergebnisse: Die Verordnungen konnten in fünf Themen kategorisiert werden. Die Daten zeigen, dass „Besuchsverbote“ (98,3\%) und „Körperkontaktreduzierung“ (90,5\%) den größten Teil der Einschränkungen darstellen. Über alle Angebote hinweg waren „Verbotsmaßnahmen“ und für viele Angebote „Kontaktreduzierungen“ bedeutsam assoziiert, während etwa der Wegfall von „Gruppenangeboten“ zweimal so hoch war, wenn die Schutzmaßnahme „Reduzierung des Körperkontaktes“ umgesetzt wurde. „Besuchseinschränkungen“ zeigten kaum signifikante Assoziationen. Schlussfolgerungen: Die Ergebnisse belegen einen Zusammenhang von Schutzmaßnahmen und Wegfall sozialer Angebote. Maßnahmen, die den Gesundheitsschutz mit dem Erhalt von sozialen Angeboten und Versorgung kombinieren, sollten priorisiert werden.
\end{abstract}

Schlüsselwörter: Covid-19-Schutzmaßnahmen, soziale Angebote, Gemeinschaftlichkeit, Berührungen, Wohlbefinden

\section{Covid-19 protections and social life limitations in nursing homes - Analysis of prescriptions and survey data}

Abstract: Background: The tension between health protection and restrictions of social life in nursing homes during the pandemic has been little addressed. Aim: The aim of the study was to systematize state-specific regulations and actual prioritizations as well as implementations of the pandemic measures and to relate them to life changes for residents. Methods: In a mixed-methods design, 450 protective measures for nursing homes nationwide were categorized and fed into a survey of nursing home managers $(n=1,260)$ on the handling of these measures in a protective measures model. The association of protective measures and limitations of social services in nursing homes was analyzed with binary logistic generalized estimation equations. Results: The prescriptions were categorized into five themes. Primary data show that "visitation bans" (98.3\%) and "physical contact reduction" (90.5\%) represented the largest proportion of restrictions. The regulations were variously associated with the "restriction of social services". Thus, across all offers prohibitions and for most offers "contact reductions" were significantly associated, for example that giving up "group opportunities" was almost two times as high when the protective measure "reduction of physical contact" was implemented. "Visit restrictions" on the other hand showed little significant association. Conclusions: The results provide evidence of an association between protective measures and social restrictions in the care homes during the pandemic. However, as these lost exchanges are of high value for the residents, aversive long-term effects must be assumed.

Keywords: COVID-19 protective measures, social services, communality, touch, well-being 
Was ist zu der Thematik schon bekannt?

Die Covid-19-Pandemie hat das Leben von Bewohner_innen in Pflegeheimen erschwerend gewandelt.

\section{Was ist neu?}

Verschiedene erlassene Schutzmaßnahmen zeigen einen Zusammenhang mit dem Wegfall von diversen sozialen Angeboten auf.

Welche Konsequenzen haben die Ergebnisse für die Pflegepraxis?

Schutzmaßnahmen - auch unabhängig einer Pandemie, die den Gesundheitsschutz der Bewohner_innen mit dem Erhalt von sozialen Angeboten und Versorgung kombinieren, sollten priorisiert werden.

\section{Einleitung}

Zum Schutz der Gesundheit von Bewohner_innen in Pflegeheimen sind mit Ausbreitung der Covid-19-Pandemie Infektionsschutzverordnungen und im Rahmen dieser, spezifische Schutzmaßnahmen erlassen worden. Ex abrupto hat sich damit das Leben von 818317 Heimbewohner_innen deutschlandweit erschwerend gewandelt (Pflegestatistik, 2019). So wurde der Besuch von Angehörigen und Freund_innen eingeschränkt oder verboten. Hygieneschutzmaßnahmen wurden ebenso umgesetzt wie Kontaktreduzierungen. Gemeinsame Veranstaltungen wurden nahezu komplett aufgehoben. Diese übergangslosen Veränderungen hatten für die Bewohner_innen erhebliche Auswirkungen. Neben gesundheitlichen Einschränkungen (Schweickert et al., 2021) - einer ohnehin vulnerablen Personengruppe - befand sich das soziale Leben in einer nahezu vollständigen Abschirmung nach Außen und Innen (BASGO, 2020), wo bereits vor der Pandemie schwierige Bedingungen und Einschränkungen im sozialen Leben in den Pflegeheimen beschrieben wurden (Trybusińska \& Saracen, 2019). Ferner erschwerten die Menge der Schutzmaßnahmenverordnungen, die Geschwindigkeit der Erlasse neuer Verordnungen und die Änderung bestehender sowohl den Bewohner_innen, den Angehörigen als auch den Pflegeheimleitungen und dem Pflegepersonal neben einer transparenten Darstellung und Umsetzung der Schutzmaßnahmen auch zu einem erheblichen Anstieg der Arbeitsanforderungen und damit zu steigenden Belastungen (BIVA, 2021). Ein inner- und außereuropäischer Blick zeigt weltweit die Aufhebung sozialer Angebote in der Heimversorgung und die Folgen von sozialer Isolation auf. Für Irland beschreiben Cathaoir und Rognlien (2021), dass eine Politik des "Cocooning“ zu Einsamkeit und Isolation führe (S. 84). Für Italien umschreiben es Trabucchi und Leo (2020) mit dem Titel „Nursing homes or besieged castles“. In einer niederländischen Querschnittsstudie gaben $77 \%$ der Bewohner_innen an, Einsamkeitsgefühle zu verspüren, weiterhin sanken Indikatoren von Wohlbefinden und Familienmitglieder berichteten über Traurigkeit bei den Bewohner_innen (Van der Roest et al., 2020). Im Rahmen einer Übersichtsarbeit von Chu, Donato-
Woodger und Dainton (2020) werden die Besuchsbeschränkungen und der Wegfall von sozialen Aktivitäten als enorme Herausforderungen vor allem für die Bewohner innen beschrieben. Trotz der internationalen Befundlage fehlt es an einer systematischen Aufarbeitung des $\mathrm{Zu}-$ sammenhangs zwischen den Schutzmaßnahmen in den Pflegeheimen und den Einschränkungen des sozialen Lebens sowie deren Folgen in Deutschland. Eine Untersuchung der sozialen Implikationen der Schutzmaßnahmen ist daher dringend notwendig für die Untermauerung der wissenschaftlichen Aufarbeitung der Lage deutscher Pflegeeinrichtungen während der Covid-19-Pandemie, aber vor allem auch für die Zeit nach der Pandemie. Die möglichen Ergebnisse können den bereits bestehenden Handlungsdruck festigen und sollen die Assoziation politischer Entscheidungen mit sozialen Einschränkungen im Setting Pflegeheim quantifizieren und deutlich machen.

\section{Ziel und Fragestellung}

Ziel dieser Studie ist es, die Priorisierungen und Umsetzungen der Schutzmaßnahmen durch die Heime herauszuarbeiten und in Verbindung mit sozialen Angeboten bzw. deren Wegfall in der Pandemiezeit zu bringen. Folgende Fragestellungen werden bearbeitet:

Können die Schutzmaßnahmen in den erlassenen Verordnungen kategorisiert und hinsichtlich ihres Einflusses auf das soziale Leben der Bewohner_innen analysiert werden?

Gibt es einen Zusammenhang zwischen den unterschiedlichen Schutzmaßnahmen und den Einschränkungen sozialer Angebote in den Heimen?

\section{Methoden}

\section{Stichprobe}

Die vorliegende Untersuchung ist Teil des Kooperationsprojektes Covid-Heim der Charité - Universitätsmedizin Berlin und dem Wissenschaftlichen Institut der AOK (WIdO) (Kohl et al., 2021, sowie eine Serie von ErgebnisReporten zum Projekt: Hering et al., 2021a, 2021b, 2021c; Dullin \& Hartwig, 2021). Die übergeordneten Forschungsfragestellungen zum Covid-Heim-Projekt, die vorab im Antrag festgelegt wurden, finden sich auf der Homepage (https://medizinsoziologie-reha-wissenschaft.charite.de/ forschung/alternsforschung/covid_heim/). In dieser werden verschiedene Analysestränge miteinander kombiniert, um Lehren aus der Pandemie für strukturelle Entwicklungen im Pflegeheimbereich ziehen zu können.

\section{Studiendesign}

Für die vorliegende Arbeit wurden die Datenbanken mit gesetzlichen Verordnungen und die Primärdaten aus der 
Heimleitungsbefragung verwendet. Im Rahmen eines dreischrittigen Mixed-Methods-Design sind die genannten Fragestellungen eruiert worden.

\section{Schritt 1: Dokumentenanalyse Verordnungen}

Basierend auf der Sammlung von über 450 erlassenen Infektionsschutzverordnungen während der ersten Welle der Covid-19-Pandemie (01. März-30. Juni 2020) sind zentrale Schutzmaßnahmen für Pflegeheime bundesweit herausgearbeitet und thematisch in Ober- und Unterkategorien in Anlehnung an die inhaltliche Strukturierung nach Mayring kategorisiert worden (Mayring \& Fenzel, 2019). Anschließend sind diese in einem Zeitstrahlmodell (Kalenderwochen) zusammenfassend eingearbeitet und für nachfolgende quantitative Analysen kodiert worden. Die gewonnenen Kategorien wurden auch für die Erstellung der Primärdatenerhebung verwendet (siehe Messungen).

\section{Schritt 2: Primärdatenerhebung}

Im Rahmen der Primärdatenerhebung bei Heimleitungen wurden alle Pflegeheime in Deutschland aus dem AOK Pflege-Navigator (Datenbank mit Informationen über Pflegeeinrichtungen) mit insgesamt 15380 Pflegeheimen (Vollerhebung), davon 11317 vollstationär, angeschrieben, die über eine E-Mail-Adresse verfügten ( $n=10026)$. All denjenigen ohne E-Mail-Adresse wurden postalische Teilnahmeeinladungen mit dem Studienlink und einem QR-Code für den Zugang zur Teilnahme zugeschickt ( $\mathrm{n}=1291)$. Zur Erhöhung der Rücklaufquote sind einmalige Erinnerungs-Emails 14 Tage nach der ersten E-Mail versendet worden. Im Zeitraum von 01. November 202028. Februar 2021 wurden über die Erhebungsplattform REDCap (Research Electronic Data Capture der Charité / $\mathrm{BIH})$ die halbstandardisierten Fragebögen online versendet. Die Antworten sollten die Entwicklungen sozialer Angebote und Schutzmaßnahmen in den Heimen während des ersten Lockdown (01. März-30. Juni 2020) deutschlandweit beschreiben. Es wurden insgesamt N = 1973 Fragebögen aufgerufen. Davon sind $\mathrm{n}=886$ vollständig $(44,9 \%)$ und $n=1087$ (55,1\%) unvollständig ausgefüllt worden. Zu welchem Zeitpunkt ein Abbruch erfolgte kann nicht nachverfolgt werden. Für die vorliegende Untersuchung wurden somit alle Fragebögen ausgewertet, die bis einschließlich der relevanten Themenblöcke zu den Schutzmaßnahmen und der sozialen Angebote ausgefüllt wurden. Grundlage der Auswertung waren somit n = 1260 Fragebögen von Heimleitungen aus ganz Deutschland. Für die Primärdaten fand eine Untersuchung der priorisierten Schutzmaßnahmen im Rahmen der Befragung von Heimleitungen deutschlandweit sowie die Befragung nach sozialen Angeboten in den Pflegeheimen während der Pandemie und deren Einschränkung statt.

\section{Schritt 3: Integration von Primärdaten und Dokumentenanalyse}

Die erlassenen Verordnungen wurden im Rahmen der Dokumentenanalyse gesetzlicher Verordnungen und die von den Heimleitungen berichteten Maßnahmen und Umsetzungen gegenübergestellt und zur statistischen Analyse in einem ausgearbeiteten Schutzmaßnahmenmodell modifiziert. Dabei stand die Analyse des Zusammenhanges von priorisierten Schutzmaßnahmen im Rahmen des Schutzmaßnahmenmodells mit Indikatoren gemeinschaftlichen, sozialen Lebens in den Pflegeheimen im Fokus.

\section{Ethische Aspekte}

Die Datenerhebung wurde mit Zustimmung der Ethikkommission (EA1/254/20 400146), mit nationalem Recht sowie der Deklaration von Helsinki 1975 durchgeführt. Alle Heimleitungen erhielten Informationen über Studienziele und wurden über die Möglichkeit des Befragungsabbruchs informiert. Von allen Heimleitungen liegt eine Einverständniserklärung vor.

\section{Messungen}

\section{Schutzmaßnahmen}

Die Umsetzung der Schutzmaßnahmen wurde im Rahmen der Primärdatenerhebung durch die von den Autor_innen entwickelten Items erfasst: „Wir würden Sie bitten in der nachfolgenden Liste anzuklicken, welche Maßnahmen von Ihnen im Zeitraum vom 01. März-30. Juni 2020 umgesetzt und / oder wieder aufgehoben werden konnten"; 4 kategoriale Antwortvarianten: 1 = Maßnahme wurde umgesetzt, 2 = Maßnahme wurde zunächst umgesetzt und dann teilweise wieder aufgehoben, 3 = Maßnahme wurde umgesetzt und dann vollständig wieder aufgehoben, $0=$ nicht zutreffend (Nicht zutreffend beschreibt, dass Schutzmaßnahmen nie umgesetzt wurden). Aus diesen Variablen wurden Dummyvariablen 0 = nicht zutreffend und 1 = umgesetzt gebildet. Die auszuwählenden Maßnahmen wurden theoretisch aus den Infektionsschutzverordnungen (siehe Schritt 1) sowie auf Literaturgrundlagen ausgearbeitet: Aufnahmestopp, bauliche Veränderungen, Besuchsverbote-/Einschränkungen, Verbot zusätzlicher Betreuungsangebote, Reduzierung von Körperkontakt, Ausgangsbeschränkungen, Abwesenheitsregelungen der Pflegekräfte, Isolation bei Erkrankten, Kontaktminimierung, Aufhebung der Bezugspflege, Aufhebung der Kurzzeitpflege, Aufhebung der Kurzzeitpflege zur Übernahme von Krankenhausfällen, Schulungen von Pflegekräften, Ausarbeitung eines Pandemieplanes, Individuelle Risikoabschätzung zur Ausgestaltung der Quarantäne.

\section{Angebote und Soziales Leben}

Das soziale Leben während der ersten Welle der Pandemie wurde mit folgendem Item erhoben: „Beschreiben Sie bitte durch die untenstehende Liste die Situation im Zeitraum vom 01. März-30. Juni 2020 in Ihrer Einrichtung"; 4 kategoriale Antwortvarianten: 1 = Angebot ist nicht entfallen, 2 = Angebot ist entfallen, 3 = Angebot ist entfallen, konnte aber wieder bereitgestellt werden, $0=$ nicht $\mathrm{zu}-$ 
treffend. Diese wurden ebenfalls als Dummyvariablen $0=$ nicht entfallen, 1 = entfallen codiert. Die angegeben sozialen Angebote sind auf Grundlage der Deutschen Gesellschaft für Pflegewissenschaft (DGP, 2020), der BAGSO (2020) dem BIVA-Pflegeschutzbund (2021) und den gesammelten Verordnungen in einem Vieraugenprinzip gesammelt und für diese Studie zusammengeführt worden. Dabei sind neben den Quellen der o. a. Gesellschaften / Interessenvertretungen auch deren Websites auf relevante / bekannte soziale Angebote in Pflegeheimen hin untersucht worden. Die gesammelten Angebote sind zu Oberbegriffen zusammengefasst worden. So ergaben sich bspw. aus den Angeboten (Spiele spielen, singen und tanzen) der Oberbegriff: Gemeinsame Veranstaltungen. Für eine universale Darstellung der Infektionsschutzmaßnahmen für das jeweilige Bundesland ist eine Tabelle erarbeitet worden. Hierbei stand eine entwickelte Stichwort-Suchstrategie der umzusetzenden Infektionsschutzmaßnahmen sowie der sozialen Angeboten innerhalb der Dokumente im Vordergrund. Schließlich sind die gesammelten Oberbegriffe mit den in den Dokumenten benannten sozialen Angeboten abgeglichen und ggf. erweitert/angepasst worden: Gemeinsame Veranstaltungen, Gemeinsames Essen, Kreative Angebote, Spaziergänge, Gottesdienste, Gedächtnistraining, Entspannungsübungen, Mobilitätstraining, Ergotherapie, Physiotherapie, Logopädie, Podologie, Individuelle medizinisch, pflegerische, seelsorgerische und/ oder psychosoziale Angebote sowie BVP-Behandlung im Voraus planen.

\section{Heimgröße}

Auf Grundlage der Frage ,Wie viele Pflegebedürftige leben aktuell in Ihrer Einrichtung?" sind Kategorien in kleine
(<50 Bewohner_innen) mittelgroße (51-100 Bewohner innen) und große Heime (> 101 Bewohner_innen) gebildet worden (TNS Infratest Sozialforschung, 2015).

\section{Statistische Analysen}

Für die Modellierung des Zusammenhangs der dummykodierten Schutzmaßnahmen (Aufnahmestopp, Besuchsverbote-/Einschränkungen, Verbot zusätzlicher Angebote, Reduzierung Körperkontakt, Isolation, Kontaktminimierung, Individuelle Risikoabschätzung) und der dummykodierten sozialen Angebote (Gemeinsame Gruppenveranstaltungen, Spaziergänge, Kreative Angebote, Entspannungsangebote, Individuelle Angebote) wurde für jede Schutzmaßnahme ein multiples logistisches Regressionsmodell mittels verallgemeinerter Schätzgleichung (Generalized Estimating Equations (GEE), Verteilung = binomial, Verknüpfungsfunktion = logit) gerechnet, in dem Heimgröße als Kovariate sowie die sozialen Angebotsvariablen eingefügt wurden. Ferner erlaubt GEE eine Adjustierung für die verschachtelte Kovarianzstruktur (= Exchangeable, Annahme gleicher Kovarianzen) der Heimleitungen / Pflegeheime der Bundesländer. Es wurde die Chance für das Eintreten des Wegfallens von sozialen Angeboten bedingt durch die jeweils umgesetzte Schutzmaßnahme geschätzt. Unter Einbezug des Hosmer- und Lemeshow-Anpassungstests wurde die Angemessenheit des Modells als Voraussetzung beurteilt (Annahme $\mathrm{p}=$ statistisch nicht signifikant). Signifikante Unterschiede sind bei einem Signifikanzniveau von $\mathrm{p}<0,05$ angenommen worden. Für die Analysen wurde SPSS v26 verwendet.

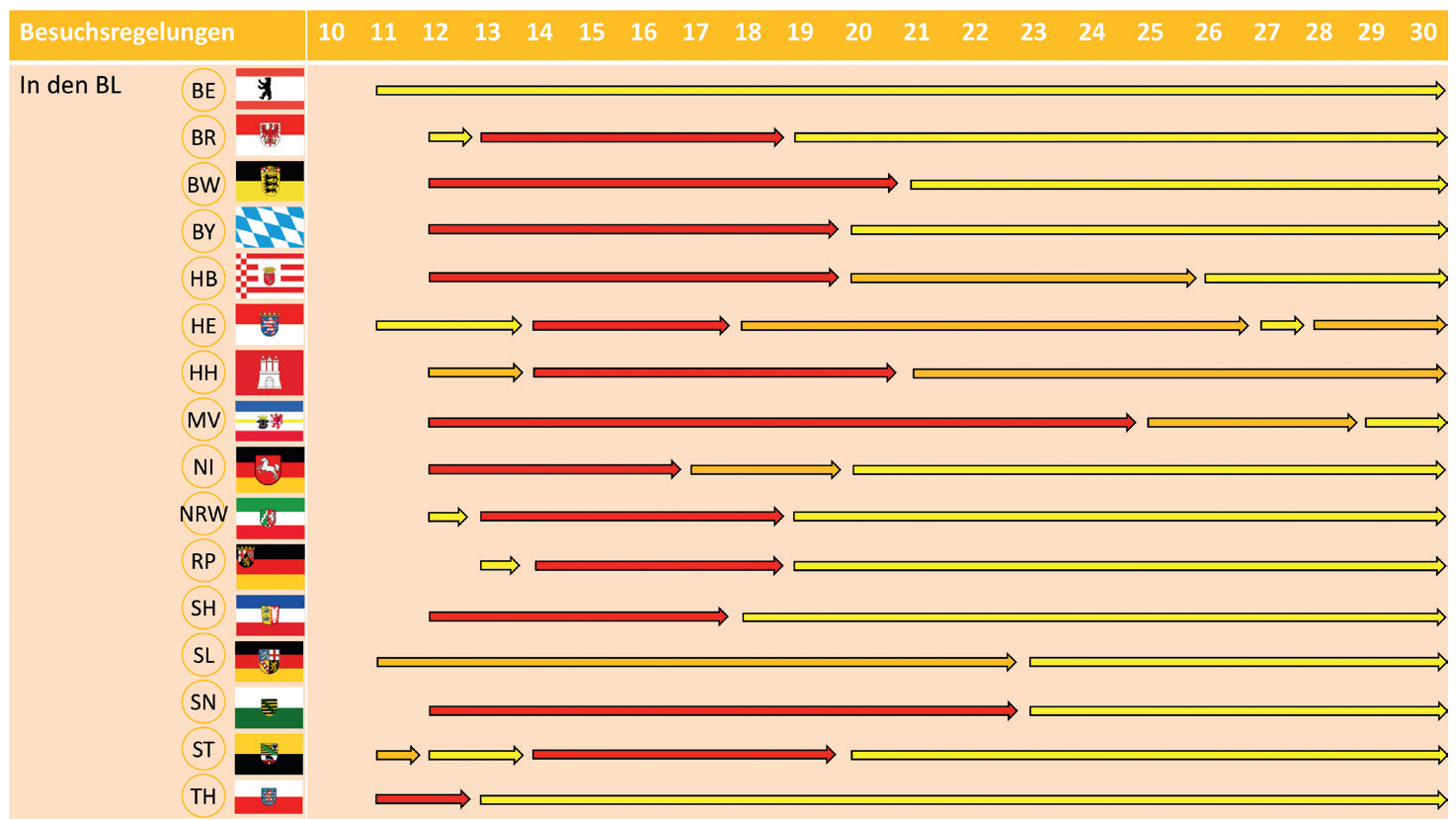

Abbildung 1. Besuchsregelungen während der ersten Welle der Covid-19-Pandemie in den Kalenderwochen 10 - 30 (01.03.2020 - 30.06.2020). BL: Bundesländer, BE: Berlin, BR: Brandenburg, BW: Baden-Württemberg, BY: Bayern, HB: Bremen, HE: Hessen, HH: Hamburg, MV: Mecklenburg-Vorpommern, NI: Niedersachsen, NRW: Nordrhein-Westfalen, RP: Rheinland-Pfalz, SH: Schleswig-Holstein, SL: Saarland, SN: Sachsen, ST: SachsenAnhalt, TH:Thüringen. Rot: Besuchsverbot; Orange: Strenge Besuchseinschränkungen, Gelb: Weniger strenge Besuchseinschränkungen, Grün: Besuche ohne Einschränkungen. 


\section{Ergebnisse}

\section{Schritt 1 Dokumentenanalyse Verordnungen}

Aus der bundesweiten Analyse der Verordnungen heraus können wir festhalten, dass deutschlandweit eine Intransparenz herrscht. Die Bundesländer haben vermehrt differente Schutzmaßnahmen in den Verordnungen festgehalten. Für die Herstellung einer Kongruenz konnten vier Oberkategorien von Schutzmaßnahmen - bestehend aus verschiedenen Unterkategorien - gebildet werden. Die Oberkategorien können in nachfolgende Themenbereiche gegliedert werden: Besuchsregelungen, Zugangsregelungen, Hygiene- und Schutzregelungen, Arbeitsleben und Soziales. Aus diesen Kategorien lassen sich die am häufigsten vertretenden Schutzregelungen deutschlandweit herausarbeiten und in einem entwickeltem „Schutzmaßnahmenuntersuchungsmodell“ hinsichtlich ihres Einflusses auf die soziale Teilhabe im Heim zusammenfassen: Aufnahmestopp, Besuchsverbote-/Einschränkungen, Reduzierung von Körperkontakt von Allen im Pflegeheim lebenden, Kontaktminimierung zwischen Pflegekräften und Bewohner_innen, Verbot / Einschränkung zusätzlicher Angebote, Isolation von Erkrankten Bewohner_innen, Individuelle Risikoabschätzung.

Entlang dieser Kategorien sind bundesweite zusammenfassende Zeitstrahlmodelle ausgearbeitet worden. Abbildung 1 zeigt das Zeitstrahlmodell für die „Besuchsregelungen“(Kategorie „Besuchsverbote-/Einschränkungen“). Tabelle 1 gibt die jeweiligen ausgearbeiteten Unterkategorien an. Weitere Zeitstrahlmodelle auf Bundeslandebene können im Elektronischen Supplement ESM1 eingesehen werden.

\section{Schritt 2 Primärdatenerhebung}

Deskriptive Ergebnisse: Nordrhein-Westfalen (21,8\%), Bayern (17,6\%), Baden-Württemberg (17,1\%) und Niedersachsen $(11,2 \%)$ weisen die größte Rücklaufquote auf. Am geringsten vertreten sind Bremen und Saarland (beide $0,9 \%)$. Jedes zweite an der Befragung beteiligte Heim hat einen freigemeinnütziger Träger (50,2\%), 40,7\% befinden sich in privater und 8,3\% in öffentlicher Trägerschaft. Mittelgroße Heime sind am häufigsten vertreten (48,9\%). Nahezu jedes vierte Heim (23,1\%) ist als groß und $28 \%$ sind als klein einzustufen (siehe Tab. 2).

Die Primärdaten in Tabelle 2 zeigen, dass „Besuchsverbote- / Einschränkungen“ mit 98,3\% und ,Körperkontaktreduzierung" zwischen allen im Heim lebenden Personen mit 90,5\% den größten Bereich ausgeführter Schutzmaßnahmen einnehmen. Mit 72,4\% ist die „Individuelle Risikoabschätzung “ für die Ausgestaltung der Quarantäne (wann, wie lange) angegeben. 64,8\% der Heimleitungen geben an, „zusätzliche Betreuungsangebote“ (zum Beispiel Vorlesen) aufgehoben zu haben. „Aufnahmestopp“ $(59,8 \%)$ und „Kontaktminimierung "zwischen dem Pflegepersonal und den Bewohner_innen geben rund $60 \%$ an.
Tabelle 1. Unterkategorien der Besuchsregelungen und deren Bedeutungen

\begin{tabular}{|c|c|}
\hline $\begin{array}{l}\text { Rot } \\
\text { Besuchsverbote }\end{array}$ & $\begin{array}{l}\text { - Verbot von Besuchen durch Angehörige } \\
\text { (sowohl in Bewohnerzimmern als auch } \\
\text { in Besuchsräumen) } \\
\text { - Nicht enthalten: Ausnahmen } \\
\text { zur Sterbebegleitung } \\
\text { - Besuchsverbot bei Covid-Fall, } \\
\text { Besucher_innen mit Atemwegs- } \\
\text { erkrankungen/Symptomen, aus Risiko- } \\
\text { gebieten, Kontaktpersonen, Kinder unter } \\
\text { 16, Kinder unter 14 Jahren }\end{array}$ \\
\hline $\begin{array}{l}\text { Orange } \\
\text { Strenge Besuchs- } \\
\text { einschränkungen }\end{array}$ & $\begin{array}{l}\text { - In Begleitung des Personals, } 45 \text { Minuten, } \\
\text { einmal/Woche, immer gleiche Person, } \\
1 \text { Person pro Woche für } 1 \text { Stunde } \\
\text { - } 2 \text { Personen für } 3 \text { Stunden } 1 \times \text { pro Woche } \\
\text { - } 1 \text { Person mindestens } 45 \text { min } 2 \times \text { pro Woche } \\
\text { - } 3 \text { Besuche pro Woche } \\
\text { - } 1 \text { Person (immer dieselbe) pro Tag mit } \\
\text { weiteren Regelungen durch das Heim } \\
\text { - } 2 \text { Personen, } 2 \times \text { pro Woche }\end{array}$ \\
\hline $\begin{array}{l}\text { Gelb } \\
\text { Weniger Strenge } \\
\text { Besuchseinschärnkungen }\end{array}$ & $\begin{array}{l}\text { - } 1 \text { Personen pro Tag für } 1 \text { Stunde } \\
\text { - } 2 \text { Personen pro Tag für } 1 \text { Stunde } \\
\text { - } 1 \text { Person pro Tag für } 2 \text { Stunden } \\
\text { - } 2 \text { Personen pro Tag für } 2 \text { Stunden } \\
\text { - Begleitung zur besuchten Person } \\
\text { durch Personal, } 2 \text { Stunden pro Tag } \\
\text { - Besuch für mindestens } 4 \text { Stunden } \\
\text { pro Tag über die Woche verteilt } \\
\text { - Besuch für mindestens } 4 \text { Stunden } \\
\text { - } 1 \text { Person pro Tag, } 1 \text { Person zu festen Zeiten } \\
\text { - } 1 \text { Besuch plus maximal } 1 \text { Begleitperson } \\
\text { - } 2 \text { Personen pro Tag zu festen Zeiten } \\
\text { - } 2 \text { Personen pro Tag } \\
\text { - Besuche erlaubt, wenn kein aktuelles } \\
\text { Infektionsgeschehen im Heim } \\
\text { (plus Hygienekonzept) } \\
\text { - Besuche am Wochenende/Feiertagen } \\
\text { - } \text { - Bne zeitliche Begrenzungen } \\
\text { - Besuche ohne zeitliche Begrenzung } 3 \text { Personen auf Freiflächen } \\
\text { - Besuche im Außenbereich von maximal } \\
\text { hen einrichtungsspezifisch anzupassen } \\
\text { Benohnerorientiertes Konzept }\end{array}$ \\
\hline $\begin{array}{l}\text { Grün } \\
\text { Besuche ohne } \\
\text { Einschränkungen }\end{array}$ & $\begin{array}{l}\text { - Besuche ohne Einschränkungen nach } \\
\text { Pandemieende }\end{array}$ \\
\hline
\end{tabular}

Anmerkungen: Die ausgearbeiteten Unterkategorien aus den Schutzmaßnahmenverordnungen können von den Heimen indviduell angepasst worden sein. Besuche ohne Einschränkungen sind (noch) nicht möglich.

Jede zweite Heimleitung (50,1\%) gibt die Schutzmaßnahme "Isolation bei Erkrankten“ (Abgrenzung in drei Bereiche / Kohortenversorgung: Covid-19-Fälle, Verdachtsfälle, Nicht-Fälle) an. Bei mehr als jedem zweiten Pflegeheim $(62,7 \%)$ sind "gemeinsame Gruppenveranstaltungen“ 
weggefallen. Bei 40,3\% der Heime sind „Kreative Angebote“ entfallen. „Individuelle Angebote“ wie Seelsorge oder individuelle medizinische Versorgungen sind bei 36,3\% der Pflegeheime aufgehoben worden. Mehr als jedes dritte Pflegeheim (34,4\%) hat das Angebot „Spaziergänge“ und 22,5\% die „Entspannungsangebote“ (nahezu jedes vierte Pflegeheim) aufgehoben (siehe Tab. 2).

\section{Schritt 3 Integration von Primärdaten und Dokumentenanalyse}

Assoziation von Schutzmaßnahmen und Einschränkungen sozialer Angebote: Die logistischen Regressionsmodelle (GEE) in Tabelle 3 zeigen signifikante Zusammenhänge zwischen einzelnen Schutzmaßnahmen und sozialen Angeboten. Zwischen der Schutzmaßnahme „Verbot zusätzlicher Angebote“ und nahezu allen sozialen Angeboten können bedeutsame Zusammenhänge angegeben werden ( $p<0,001)$. Die Assoziationsmaße (Odds Ratio) nehmen hierbei alle Werte über 1 an („Gruppenangebote“ $O R=2,16$, 95\%-KI $[1,66 ; 2,84]$; „Spaziergänge“ OR = 4,14, $95 \%-\mathrm{KI}$
$[3,10 ; 5,55]$; „Kreative Angebote“ OR = 2,96, 95\%-KI [2,32; $3,78]$; „Entspannungsangebote“ $\mathrm{OR}=3,28,95 \%-\mathrm{KI}[2,56$; 4,21]; „Individuelle Angebote“ $\mathrm{OR}=1,75,95 \%-\mathrm{KI}[1,23$; $2,49])$. So ist die Chance des Wegfalls mit Umsetzung dieser Schutzmaßnahme bei den „Spaziergängen“ um das Vierfache erhöht, bei den „Individuellen Angeboten“ (beispielsweise der Seelsorge) beträgt die Wegfallchance 75,7\%. Das Risiko ist zweimal höher das „Gruppenveranstaltungen" aufgehoben werden, wenn die Maßnahme „Reduzierung des Körperkontaktes" zwischen allen Personen umgesetzt wird ( $\mathrm{OR}=2,37,95 \%$-KI $[1,46,3,85]$, $\mathrm{p}<0,001)$. Die Chancenverhältnisse zur Aufhebung zwischen der Maßnahme „Kontaktminimierung zwischen dem Pflegepersonal und den Bewohner_innen" und den sozialen Angeboten sind signifikant und ergeben positive Assoziationsmaße (OR >1). Die soziale Aktivität der „Kreativen Angebote" ist hierbei mit einer zweifachen Wegfallchance signifikant $(\mathrm{OR}=2,03,95 \%-\mathrm{KI} \quad[1,54 ; 2,69]$, $\mathrm{p}<0,001)$. Eine $86 \%$-ige Chance des Wegfalls besteht bei den „Entspannungsangeboten“ (OR = 1,86, 95\%-KI [1,26; 2,75], p = 0.002). Die „Besuchseinschränkungen“ zeigen nur mit den „Entspannungsangeboten“ signifikante Asso-

Tabelle 2. Überblick nach Bundesländern, Trägerschaft, Heimgröße, Anteil der umgesetzten Schutzmaßnahmen und entfallenen sozialen Angebo$\operatorname{ten}(n=1260)$

\begin{tabular}{|c|c|c|c|c|c|}
\hline Bundesländer & Anteil (\%) & Trägerschaft & Anteil (\%) & Vgl. Pflegestatistik 2019 (\%) & \\
\hline Bayern & 17,6 & Öffentlicher Träger & 8,3 & 4,5 & \\
\hline Bremen & 0,9 & Freigemeinnütziger Träger & 50,2 & 52,7 & \\
\hline Baden-Württemberg & 17,1 & Privater Träger & 40,7 & 42,7 & \\
\hline Brandenburg & 3,7 & Gesamt & 99,3 & 99,9 & \\
\hline Berlin & 4,4 & Fehlende Angaben & 0,7 & 0,1 & \\
\hline Hessen & 6,5 & Heimgröße & & & \\
\hline Hamburg & 2,2 & Klein (1 - 50 Plätze) & 28 & 47,4 & \\
\hline $\begin{array}{l}\text { Mecklenburg } \\
\text { Vorpommern }\end{array}$ & 3,3 & Mittel (51 - 100 Plätze) & 48,9 & 36,9 & \\
\hline Nordrhein-Westfalen & 21,8 & Groß (101< Plätze) & 23,1 & 15,7 & \\
\hline Niedersachsen & 11,2 & Schutzmaßnahmen & $\begin{array}{l}\text { Anzahl } \\
\text { umgesetzt (\%) }\end{array}$ & Soziale Angebote & $\begin{array}{l}\text { Anzahl } \\
\text { entfallen (\%) }\end{array}$ \\
\hline Rheinland-Pfalz & 3,5 & Aufnahmestopp ( $n=1001)$ & 59,8 & $\begin{array}{l}\text { Gemeinsame Gruppenveranstal- } \\
\text { tungen }(n=1004)\end{array}$ & 62,7 \\
\hline Schleswig-Holstein & 4,5 & Besuchsverbote $(n=1045)$ & 98,3 & & \\
\hline Sachsen & 4,2 & Verbot zusätzlicher Angebote $(n=1034)$ & 64,8 & $\begin{array}{l}\text { Kreative Angebote (z.B. basteln, } \\
n=1005)\end{array}$ & 40,3 \\
\hline Sachsen-Anhalt & 3,4 & Reduzierung Körperkontakt ( $\mathrm{n}=1042$ ) & 90,5 & $\begin{array}{l}\text { Entspannungsangebote (z.B. auto- } \\
\text { genes Training, } n=976 \text { ) }\end{array}$ & 22,5 \\
\hline Saarland & 0,9 & Isolation ${ }^{b}(n=1023)$ & 50,1 & $\begin{array}{l}\text { Individuelle Angebote (medizinisch, } \\
\text { pflegerisch, seelsorgerisch, BVPc, } \\
n=984 \text { ) }\end{array}$ & 36,3 \\
\hline Thüringen & 2,8 & $\begin{array}{l}\text { Kontaktminimierung zwischen Pflege- } \\
\text { personal und Bewohner_innen }(n=1053)\end{array}$ & 59,9 & Spaziergänge ( $n=1017)$ & 34,4 \\
\hline Gesamt & 99,9 & $\begin{array}{l}\text { Individuelle Risikoabschätzung } \\
(n=1033)\end{array}$ & 72,4 & & \\
\hline Fehlende Angaben & 0,1 & & & & \\
\hline
\end{tabular}


ziationen $(\mathrm{p}=0,006)$, allerdings mit einem OR unter 1 (OR = 0,04, 95\%-KI [0,006; 0,42]). Die Maßnahme der „Isolation von Erkrankten" weist eine statistische Signifikanz mit den "Individuellen Angeboten“ auf $(\mathrm{OR}=1,5,95 \%$-KI $[1,19 ; 1,93]$, $\mathrm{p}<0,001)$. Die angezeigten Koeffizienten für die Heimgröße „klein“ und „mittel“ sind im Verhältnis zur Referenzkategorie "groß" zu sehen. Kleine und mittelgroße Heime unterscheiden sich bei den Angeboten „Gemeinsame Gruppenveranstaltungen" (beide Heimgrößen p < 0,001), "Kreative Angebote" (beide Heimgrößen $\mathrm{p}<0,001$ ), und „Entspannungsangebote“ (klein $\mathrm{p}<0,001$; mittel $\mathrm{p}=0,014$ ) signifikant von großen Heimen.

\section{Diskussion}

Die vorliegende Teiluntersuchung des Projekts Covid-Heim zeigt erstmals für Deutschland auf, dass Maßnahmen zum Schutz der Gesundheit der Heimbewohner_ innen eine Aufhebung sozialer Angebote bedingen und wie hoch eine Aufhebung mit Schutzmaßnahmen assoziiert werden können. Zudem wird ein Überblick über den Wegfall sozialer Angebote während der Pandemie in deutschen Pflegeheimen sowie eine zeitliche Übersicht der Umsetzung von Schutzmaßnahmen gegeben, die so noch nicht vorliegt. Die Dokumentenanalyse gesetzlicher Verordnungen zeigt, dass in Deutschland respektive in den Bundesländern sehr unterschiedliche Schutzmaßnahmen zu teilweise unterschiedlichen Zeitpunkten umgesetzt worden. Durch den Erlass von Allgemeinverfügungen und/oder Hygieneplänen wurden zudem zwischen einzelnen Einrichtungen sehr unterschiedlich ausgelegte Maßnahmen umgesetzt, was zusätzliche Heterogenität zwischen den Pflegeeinrichtungen bedeutet, die in der vorliegenden Arbeit nicht abgebildet ist und die in zukünftiger Forschung zusätzlich beachtet werden sollte. Der BIVA-Pflegeschutzbund forderte daher einheitliche Regelungen, die der Intransparenz entgegenwirken (BIVA, 2021). Der Wegfall sozialer Angebote durch Schutzmaßnahmen wurde bisher nicht untersucht, allerdings können die Ergebnisse in Beziehung zu Studien aus Kanada (Chu et al., 2020), Italien (Trabucchi \& Leo, 2020), den Niederlanden (Verbeek et al., 
2020) oder China (Sun \& Lu, 2020), in denen über die Ausmaße der Isolation durch Besuchseinschränkungen und/oder den eingeschränkten/entfallenen Veranstaltungen während der ersten Pandemiewelle berichtet wird, gesetzt werden. Besonders hervorgehoben werden sollte die pandemische Krisensituation in Italien. Trabucchi und Leo (2020) berichten über die ausweglose Situation für alle Beteiligten, besonders aber für die Bewohner_innen. So sind gleichermaßen sowohl in Deutschland als auch in Italien schlagartig soziale Angebote aufgehoben und Isolationsmaßnahmen ergriffen worden. Ebenfalls stehen die Ergebnisse im Einklang mit der Arbeit von Hämel et al. (2020), die im Rahmen leitfadengestützter Interviews mit Heimleitungen und Fachkräften berichten, dass in Folge der Schutzmaßnahmen in der ersten Pandemiewelle soziale Angebote ausgesetzt und / oder stark reduziert werden mussten. Internationale Studien weisen ähnliche Befunde auf, sowohl die Aufhebung von Gruppenaktivitäten als auch die Umstrukturierung von Veranstaltungen in kleinere Gruppen (Forma, Aaltonen \& Pulkki, 2020; Rostgaard, 2020). Mit Aufhebung und Umstrukturierung von Veranstaltungen wird zudem auf das Wohlbefinden der Bewohner_innen verwiesen, welches sich mit dem Wegfall von Routinen und sozialen Angeboten verschlechtern kann (Abbasi, 2020; Hemberg, Nyqvist, Ueland \& Näsman, 2020, Van der Roest et al., 2020). Ein festes soziales Umfeld mit engen sozialen Verbindungen ist für das Wohlbefinden eines Menschen jedoch unumgänglich (Gallagher, 2012; Drageset, Eide, Kirkevold \& Ranhoff, 2013; Neves, Sanders \& Kokanović, 2019). Die Ergebnisse einer Umsetzung von „Besuchsverboten-/ Einschränkungen“ sowie „Reduzierung des Körperkontaktes“ berichten beispielsweise auch Van der Roest et al. (2020) oder Forma et al. (2020). Dänische Pflegeheime wiesen bereits mit dem Erlass von Schutzmaßnahmen daraufhin, die Tagesstruktur weitgehend aufrechtzuerhalten, um den mentalen, physischen Zustand aktiv zu halten (Rostgaard, 2020). Sicherlich sind auch hier Einschränkungen im (sozialen) Lebensalltag der Heimbewohner_innen nicht zu vermeiden gewesen, allerdings wurde frühzeitig auf die Wichtigkeit der mentalen Gesundheit aufmerksam gemacht. So wurde in Dänemark aktiv erwähnt, dass mit Umsetzung von Schutzmaßnahmen mögliche mentale Konsequenzen von Bewohner_innen berücksichtigt werden sollen, um Folgen so früh wie möglich abzufedern. Der Appell, soziale Teilhabe in den Pflegeheimen auch während der Pandemie aufrechtzuerhalten, ist in all den genannten Veröffentlichungen sehr deutlich formuliert - auch ungeachtet der Gefahren, die eine mögliche Ansteckung bedeutet (BASGO, 2021). Unsere Ergebnisse zeigen, dass die Schutzmaßnahme „Verbot von zusätzlichen Angeboten“ signifikant den Wegfall aller angegebenen Angebote bedingen kann, dies war vor allem für „Individuelle Angebote" (u.a. Seelsorge) der Fall. Überraschend ist hingegen, dass „Besuchsverbote-/Einschränkungen“ keine Signifikanz zu sozialen Angeboten aufwiesen. Wir deuten dies als Alleinstellungsmerkmal der Besuche unabhängig von sozialen Angeboten. So befinden sich Bewohner_innen und Besuchende meistens in einem privaten Raum, um die gemeinsame Zeit intensiv zu nutzen und weniger in einer größeren Gesellschaft. Strukturell zeigt sich, dass kleine und mittelgroße Heime eine geringere Wegfallchance sozialer Angebote (Gruppenangebote, Kreative Angebote und Entspannungsangebote) durch die Schutzmaßnahmenumsetzung zu haben scheinen. Dies könnte mit der höheren Fluktuationsrate der Pflegekräfte in großen Pflegeheimen zusammenhängen (TNS Infratest Sozialforschung, 2015). Das wechselnde Personal könnte eine geringere emotionale Verbundenheit zu den Bewohner_innen aufgebaut haben, sodass die sozialen Angebote aufgrund einer möglichen höheren Anonymität schneller aufgehoben werden könnten. Besonders die Maßnahme der „Körperkontaktminimierung“ weist mit allen sozialen Angeboten signifikante Zusammenhänge auf. Hierbei vermuten wir, dass nicht nur der kurzzeitige zwischenmenschliche Kontakt von Bedeutung ist, sondern vor allem „affektive Berührungen“. Berührungen finden bei allen Angeboten statt. Sie sind essenziell - Emotionsregulation, Entspannung, Stärkung des Wohlbefindens und der Immunabwehr sind positive Effekte (Cohen, JanickiDeverts, Turner \& Doyle, 2015; Helmbold, 2007). Der Entzug von Berührungen durch die Maßnahmen der „Kontaktreduzierungen“, „Verbote zusätzlicher Angebote“ oder „Besuchsverbote“ können nicht nur Verschlechterungen des Wohlbefindens hervorrufen, sondern lassen auch weiterhin Einbußen des Gesundheitszustandes erwarten - vor allem, wenn diese soziale Ausgeschlossenheit weitergeführt wird. Die Aufrechterhaltung des sozialen Lebens ist eine Notwendigkeit für die körperliche, emotionale und mentale Gesundheit sowie ein zufriedenes Leben (Hemberg et al., 2020). Ebenso weisen die Ergebnisse auf den zusätzlichen Mehraufwand für die Pflegekräfte. So mussten diese den Besuchsausfall von Angehörigen - diesen emotionalen Verlust - neben der Versorgung, kompensieren. Es könnte eine Entlastung schaffen, wenn Angehörige/Besuchende in Pflegeheime sowohl für "einfache Besuche" als auch für die Organisation von sozialen Veranstaltungen kommen. Dies zeigt, dass soziale Teilhabestrukturen nicht für die Bewohner_innen wichtig sind, sondern auch für Pflegekräfte.

\section{Stärken und Limitationen}

Die Stärken der Studie liegen in der Synthese von Dokumentenanalyse und Surveydaten als Mixed-MethodsDesign aus dem ein Schutzmaßnahmenmodell entwickelt wurde. Als Limitation der retrospektiven Befragung kann ein recall bias nicht ausgeschlossen werden, allerdings kann hervorgehoben werden, dass die abgefragten Fakten klar zu erinnern sind und sicher auch heimintern dokumentiert wurden, so dass die Heimleitungen dies validieren konnten. Die Ergebnisse beziehen sich auf den Zeitraum der ersten Welle und wir haben keine Daten vor der Pandemie, daher sehen wir nur das Ausmaß der Einsamkeit, nicht, welcher Anteil auf die Pandemie zurückführbar 
ist und welcher vorher bereits bestand. Dennoch zeigen die absoluten Zahlen der Isoliertheit während der Pandemie ein eindeutiges Bild von nicht tragbaren Teilhabeeinschränkungen, die so vor der Pandemie nicht vorgelegen haben (Trybusińska \& Saracen, 2019). Die Rücklaufquote beträgt $11 \%$ und eine Repräsentativität der Ergebnisse kann somit nicht garantiert werden. Dennoch liegt diese Rücklaufquote im Rahmen vergleichbarer Studien (WolfOstermann et al., 2020) und der Vergleich mit der Pflegestatistik lässt zumindest für die Merkmale Heimgröße, Trägerschaft und Bundesland eine Vergleichbarkeit mit der Grundgesamtheit vermuten. Zeit ist in der Pflege eine wesentliche Ressource, sodass eine fehlende Teilnahme möglicherweise auf die Länge (ca. 20 Minuten) sowie der Freiwilligkeit zurückgeführt werden können, dies mag auch durch die hohe Abbruchquote während des Ausfüllens belegt sein, die allerdings auch typisch für OnlineSurveys ist.

\section{Schlussfolgerungen}

Die Ergebnisse zeigen eine schnelle Umsetzung der Schutzmaßnahmen und die damit verbundenen sozialen Folgen für die Bewohner_innen. Das Spannungsfeld zwischen Gesundheitsschutz und Einschränkungen des sozialen Lebens in den Pflegeheimen konnte nicht im Sinne des Bewahrens der sozialen Eingebundenheit gelöst werden und führte damit ebenfalls $\mathrm{zu}$ einer gesundheitlichen Gefährdung der Bewohner_innen, dessen Ausmaß heute noch nicht zu bemessen ist. Die Ergebnisse können von politischen Entscheidungsträgern zur Entwicklung künftiger Schutzmaßnahmen besonders mit Blick auf den möglichen Wegfall sozialer Angebote bei Umsetzung einer Schutzmaßnahme für Pflegeheime genutzt werden. Dabei gilt die Maxime, den Schutz vor Ansteckung so zu gestalten, dass soziales Leben unter anderem durch soziale Aktivitäten und Kontakte erhalten bleibt.

\section{Elektronische Supplemente (ESM)}

Die elektronischen Supplemente sind mit der OnlineVersion dieses Artikels verfügbar unter https://doi.org/10. 1024/1012-5302/a000854.

ESM1. Abbildungen zusammengefasst. Die Abbildungen zeigen die Umsetzungen der Bundesländer von weiteren Schutzmaßnahmen in einem Zeitstrahlmodell.

\section{Literatur}

Abbasi, J. (2020). Social Isolation-the Other COVID-19 Threat in Nursing Homes. JAMA, 324(7), $619-620$.

BAGSO - Bundesarbeitsgemeinschaft der Senioren-Organisationen e.V. (2020). Soziale Teilhabe in Pflegeheimen auch unter Corona Bedingungen sicherstellen. Verfügbar unter https://www.
bagso.de/fileadmin/user_upload/bagso/06_Veroeffent lichungen/2020/BAGSO-Stellungnahme_Soziale_Teilhabe_in_ Pflegeheimen_sicherstellen.pdf. 09.05.2020. [18.05.2021].

BIVA - Pflegeschutzbund (2021). Einheitliche Regelung für Pflegeheime muss Fehler der Bundesländer vermeiden BIVA-Pflegeschutzbund. Verfügbar unter https://www.biva.de/einheitlicheregelung-fuer-pflegeheime-muss-fehler-der-bundeslaendervermeiden/ [14.05.2021].

Ó Cathaoir, K., \& Gundersby Rognlien, I. (2021). The Rights of Elders in Ireland during COVID-19. European journal of health law, 28(1), $81-101$

Chu, C. H., Donato-Woodger, S. \& Dainton, C. J. (2020). Competing crises: COVID-19 countermeasures and social isolation among older adults in long-term care. Journal of Advanced Nursing, 76(10), $2456-2459$.

Cohen, S., Janicki-Deverts, D., Turner, R. B. \& Doyle, W. J. (2015). Does hugging provide stress-buffering social support? A study of susceptibility to upper respiratory infection and illness. Psychological Science, 26(2), 135 - 147.

DGP - Deutsche Gesellschaft für Pflegewissenschaft. (2020). S1 Leitlinie - Soziale Teilhabe und Lebensqualität in der stationären Altenhilfe unter den Bedingungen der COVID-19-Pandemie Langfassung - AWMF Register-nummer 184-001, Leitlinienreport. Verfügbar unter https://www.awmf.org/leitlinien/detail/ ll/184-001.html [18.06.2021].

Drageset, J., Eide, G.E., Kirkevold, M. \& Ranhoff, A. H. (2013). Emotional loneliness is associated with mortality among mentally intact nursing home residents with and without cancer: a fiveyear follow-up study. Journal of Clinical Nursing, 22, 106 - 114.

Dullin, M. \& Hartwig, S. (2021). Lehren aus der Corona-Pandemie für Strukturentwicklungen im Versorgungssetting Pflegeheim. Covid-19 assoziierte Todesfälle von Heimbewohner:innen im Spiegel der Rechtsmedizin. Verfügbar unter_https://medizinsoziologiereha-wissenschaft.charite.de/fileadmin/user_upload/ microsites/m_cc01/medizinsoziologie-reha-wissenschaft/ Dateien_Forschung/Alternsforschung/CovidHeim_ ErgebnisReport4_23_06_2021.pdf [20.08.2021].

Forma, L., Aaltonen, M. \& Pulkki, J. (2020). COVID-19 and clients of long-term care in Finland-impact and measures to control the virus. Verfügbar unter https://tccovid.org/2020/06/14/newreport-covid-19-and-clients-of-long-term-care-in-finlandimpact-and-measures-to-control-the-virus/ [14.06. 2021].

Gallagher, C. (2012). Connectedness in the Lives of Older People in Ireland: A Study of the Communal Participation of Older People in Two Geographic Localities. Irish Journal of Sociology, 20(1), $84-102$

Hämel, K., Horn, A., Rolf, A., Graffmann-Weschke, K., PetereitHaack, G., Ziech, P. \& Seidler, A. (2020). Ermöglichung sozialer Kontakte von Bewohner*innen in Alten- und Pflegeheimen während der COVID-19-Pandemie. Verfügbar unter https://www. public-health-covid19.de/images/2020/Ergebnisse/Hinder grundpapier_Heime_SozialeKontakte_201215_final.pdf [05.06.2021].

Hemberg, J., Nyqvist, F., Ueland, V. \& Näsman, M. (2020). Experiences of longing in daily life and associations to well-being among frail older adults receiving home care: a qualitative study. International Journal of Qualitative Studies on Health and Well-Being, 15(1), 1857950

Helmbold, A. (2007). Berühren in der Pflegesituation. Intentionen, Botschaften und Bedeutung (1.Aufl.). Bern: Hans Huber.

Hering, C., Gangnus, A., Kohl, R., Steinhagen-Thiessen, E. S., Sander, C. S., Gellert, P. et al. (2021a). Lehren aus der CoronaPandemie für Strukturentwicklungen im Versorgungssetting Pflegeheim. Systemrelevant und stark Belastet: Zum Befinden des Pflegepersonals in der Corona-Pandemie. Verfügbar unter https://medizinsoziologie-reha-wissenschaft.charite.de/ fileadmin/user_upload/microsites/m_cc01/medizinsoziologiereha-wissenschaft/Dateien_Forschung/Alternsforschung/Covid Heim_ErgebnisReport1_31_03_2021_neu.pdf_[17.08.2021].

Hering, C., Gangnus, A., Kohl, R., Steinhagen-Thiessen, E. S., Sander, C. S., Gellert, P. et al. (2021b). Lehren aus der CoronaPandemie für Strukturentwicklungen im Versorgungssetting 
Pflegeheim. Zur Infektions- und Versorgungssituation in der Corona-Pandemie. Verfügbar unter https://medizinsoziologiereha-wissenschaft.charite.de/fileadmin/user_upload/ microsites/m_cc01/medizinsoziologie-reha-wissenschaft/ Dateien_Forschung/Alternsforschung/CovidHeim_Ergebnis Report2_22_04_2021.pdf [17.08.2021].

Hering, C., Gangnus, A., Kohl, R., Steinhagen-Thiessen, E. S., Sander, C. S., Gellert, P. et al. (2021c). Lehren aus der CoronaPandemie für Strukturentwicklungen im Versorgungssetting Pflegeheim. Isolation und Einsamkeit: Zur Umsetzung von Schutzmaßnahmen und Folgen für Heimbewohner:innen. Verfügbar unter https://medizinsoziologie-reha-wissenschaft. charite.de/fileadmin/user_upload/microsites/m_cc01/ medizinsoziologie-reha-wissenschaft/Dateien_Forschung/ Alternsforschung/CovidHeim_ErgebnisReport3_9_06_2021_ neu.pdf [17.08.2021].

Kohl R., Jürchott K., Hering C., Gangnus A., Kuhlmey A. \& Schwinger A. (2021). COVID-19-Betroffenheit in der vollstationären Langzeitpflege. In K. Jacobs, A. Kuhlmey, S. Greß, J. Klauber \& A. Schwinger (Hrsg.). Pflege-Report 2021 (S.4-18). Springer: Berlin, Heidelberg.

Mayring, P. \& Fenzel, T. (2019). Qualitative Inhaltsanalyse. In N. Baur \& J. Blasius (Hrsg.), Handbuch Methoden der empirischen Sozialforschung (S.633 - 648, 2. Aufl.). Wiesbaden: Springer VS.

Neves, B. B., Sanders, A. \& Kokanović, R. (2019). "It's the worst bloody feeling in the world": Experiences of loneliness and social isolation among older people living in care homes. Journal of Aging Studies, 49, 74-84

Pflegestatistik. (2019). Pflege im Rahmen der Pflegeversicherung. Deutschlandergebnisse. Verfügbar unter https://www.destatis. de/DE/Themen/Gesellschaft-Umwelt/Gesundheit/Pflege/ Publikationen/Downloads-Pflege/pflege-deutschland ergebnisse-5224001199004.pdf?_-_blob=publicationFile [14.05.2021].

Rostgaard, T. (2020). The COVID-19 Long-Term Care situation in Denmark. Verfügbar unter https://ltccovid.org/wp-content/ uploads/2020/05/The-COVID-19-Long-Term-Care-situationin-Denmark-29-May-2020.pdf [08.06.2021].

Schweickert, B., Klingeberg, A., Haller, S., Richter, D., Schmidt, N., Abu Sin, M. et al. (2021). COVID-19-Ausbrüche in deutschen Alten-und Pflegeheimen. Verfügbar unter https://www.rki.de/ SharedDocs/Publikationen/DE/2021/S/Schweickert_B.html [25.06.2021].

Sun, Q. \& Lu, N. (2020). Social Capital and Mental Health among Older Adults Living in Urban China in the Context of COVID-19 Pandemic. International Journal of Environmental Research and Public Health, 17(21), 7947.

TNS Infratest Sozialforschung. (2015). Abschlussbericht - Studie zur Wirkung des Pflege-Neuausrichtungs-Gesetzes (PNG) und des ersten Pflegestärkungsgesetzes (PSG I). Verfügbar unter https://www.bundesgesundheitsministerium.de/fileadmin/ Dateien/5_Publikationen/Pflege/Berichte/Abschlussbericht_ Evaluation_PNG_PSG_I.pdf [08.06.2021].

Trabucchi, M. \& Leo, D. de (2020). Nursing homes or besieged castles: COVID-19 in northern Italy. Lancet Psychiatry 7(5), $387-388$.

Trybusińska, D. \& Saracen, A. (2019). Loneliness in the Context of Quality of Life of Nursing Home Residents. Open medicine (Warsaw, Poland), 14, 354-361.

Van der Roest, H. G., Prins, M., van der Velden, C., Steinmetz, S., Stolte, E., van Tilburg, T. G. et al. (2020). The Impact of COVID-19 Measures on Well-Being of Older Long-Term Care Facility Residents in the Netherlands. Journal of the American Medical Directors Association, 21(11), $1569-1570$.

Wolf-Ostermann, K., Schmidt, A., Preuß, B., Heinze, F., Seibert, K., Friedrich, A. C. et al. (2020). Pflege in Zeiten von Corona: Ergebnisse einer deutschlandweiten Querschnittbefragung von am- bulanten Pflegediensten und teilstationären Einrichtungen. Pflege, 33(5), 277-288.

Verbeek, H., Gerritsen, D. L., Backhaus, R., de Boer, B. S., Koopmans, R. \& Hamers, J. (2020). Allowing Visitors Back in the Nursing Home During the COVID-19 Crisis: A Dutch National Study Into First Experiences and Impact on Well-Being. Journal of the American Medical Directors Association, 21(7), 900 - 904.

\section{Historie}

Manuskripteingang: 05.07.2021

Manuskript angenommen: 19.09.2021

Onlineveröffentlichung: 13.12.2021

\section{Autorenschaft}

Konzeption des Beitrages, Erfassung, Analyse und Interpretation der Daten: AG, CH, RK, CSS, AS, EST, AK, PG

Manuskripterstellung und kritische Überarbeitung wichtiger intellektueller Inhalte: AG, CH, RK, CSS, AS, EST, AK, PG

Genehmigung der letzten Version des Manuskriptes zur Publikation: AG, CH, RK, CSS, AS, EST, AK, PG

Übernahme der Verantwortung für alle Aspekte der Arbeit: AG, CH, RK, CSS, AS, EST, AK, PG

\section{Danksagung}

Wir möchten uns bei allen Heimleitungen herzlich für die Teilnahme am Fragebogen und den zusätzlichen wertvollen Informationen bedanken.

\section{Förderung}

Das Projekt Covid-Heim: Lehren aus der Corona-Pandemie für Strukturentwicklungen im Versorgungssetting Pflegeheim wird vom Spitzenverband Bund der Krankenkassen (GKV -Spitzenverband) gefördert.

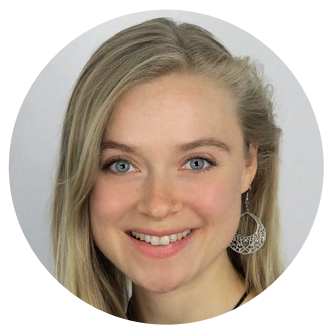

\section{Annabell Gangnus M.A.}

Institut für Medizinische Soziologie und Rehabilitationswissenschaft Charité - Universitätsmedizin Berlin Virchowweg 22 10117 Berlin Deutschland annabell.gangnus@charite.de

\section{Was war die größte Herausforderung bei Ihrer Studie?}

Strukturierung und Sammlung der sich verändernden Verordnungen.

\section{Was wünschen Sie sich bezüglich der Thematik für die Zukunft?} Dass Pandemieschutz und soziale Partizipation in allen Pflegeheimen gleichermaßen gewährleistet werden können.

\section{Was empfehlen Sie zum Weiterlesen/Vertiefen?}

Leontowitsch, M., Oswald, F., Schall, A. \& Pantel, J. (2021). Doing time in care homes: insights into the experiences of care home residents in Germany during the early phase of the COVID-19 pandemic. Ageing \& Society, $1-19$. 